\title{
Junb regulates arterial contraction capacity, cellular contractility, and motility via its target $M y / 9$ in mice
}

\author{
Alexander H. Licht,, ${ }^{1}$ Tobias Nübel,1 ${ }^{1}$ Anja Feldner, ${ }^{2}$ Nathalie Jurisch-Yaksi, ${ }^{1}$ Marco Marcello, ${ }^{3}$ \\ Elena Demicheva, ${ }^{2}$ Jun-Hao Hu, ${ }^{4}$ Bettina Hartenstein, ${ }^{1}$ Hellmut G. Augustin, ${ }^{4}$ \\ Markus Hecker, ${ }^{2}$ Peter Angel, ${ }^{1}$ Thomas Korff, ${ }^{2}$ and Marina Schorpp-Kistner ${ }^{1}$

\begin{abstract}
1Division of Signal Transduction and Growth Control (A100), German Cancer Research Center (DKFZ), DKFZ-ZMBH Alliance, Heidelberg, Germany.
Institute of Physiology and Pathophysiology, Division of Cardiovascular Physiology, University of Heidelberg, Heidelberg, Germany.

${ }^{3}$ School of Biological Sciences, University of Liverpool, Liverpool, United Kingdom. ${ }^{4}$ Joint Research Division of Vascular Biology, Medical Faculty Mannheim (CBTM), Heidelberg University and German Cancer Research Center (DKFZ-ZMBH) Alliance, Heidelberg, Germany.
\end{abstract}

\begin{abstract}
Cellular contractility and, thus, the ability to alter cell shape are prerequisites for a number of important biological processes such as cytokinesis, movement, differentiation, and substrate adherence. The contractile capacity of vascular smooth muscle cells (VSMCs) is pivotal for the regulation of vascular tone and thus blood pressure and flow. Here, we report that conditional ablation of the transcriptional regulator Junb results in impaired arterial contractility in vivo and in vitro. This was exemplified by resistance of Junb-deficient mice to DOCA-salt-induced volume-dependent hypertension as well as by a decreased contractile capacity of isolated arteries. Detailed analyses of Junb-deficient VSMCs, mouse embryonic fibroblasts, and endothelial cells revealed a general failure in stress fiber formation and impaired cellular motility. Concomitantly, we identified myosin regulatory light chain 9 (My19), which is critically involved in actomyosin contractility and stress fiber assembly, as a Junb target. Consistent with these findings, reexpression of either Junb or Myl9 in Junb-deficient cells restored stress fiber formation, cellular motility, and contractile capacity. Our data establish a molecular link between the activator protein-1 transcription factor subunit Junb and actomyosin-based cellular motility as well as cellular and vascular contractility by governing Myl9 transcription.
\end{abstract}

\section{Introduction}

Cardiovascular diseases are linked with a number of pathological conditions and nowadays still constitute the major cause of death globally. Aberrant regulation of vascular tone plays an important role in the pathophysiology of major cardiovascular diseases including hypertension, myocardial infarction, and atherosclerosis (1-3). A primary determinant of vascular tone, hence resistance and regional blood flow, is the contractile capacity of VSMCs. Abnormal contractility of VSMCs coincides with hypertension and vasospasm superimposed on atherosclerosis (4). Several layers of VSMCs compose the wall of arterioles and in particular conduit arteries, while small capillaries and lymphatic vessels are covered by pericytes, a subtype of specialized contractile cells $(5,6)$. Both pericytes and VSMCs express non-muscle myosin and $\alpha$-SMA and have thick actin stress fibers (6). They represent the main components of the contractile machinery of these cells and thus are implicated in cell contraction and relaxation. Actin stress fibers that are connected at both ends to focal adhesions transmit the contractile force required for actomyosin-mediated contraction. Pressure- or volume-induced distention as well as active relaxation of the vessel wall lead to stretching of ECs and, in particular, of VSMCs, with subsequent induction of stress fiber formation $(6,7)$. This, in turn, helps the vessel wall to maintain its tone and the perfusion of the downstream tissue, whereas excessive or abnormal contractility

Authorship note: Alexander H. Licht and Tobias Nübel contributed equally to this work.

Conflict of interest: The authors have declared that no conflict of interest exists. Citation for this article: J Clin Invest. 2010;120(7):2307-2318. doi:10.1172/JCI41749. contributes to hypertension or vasospasm and, consequently, to ischemia, especially in coronary and cerebral arteries $(6,8,9)$.

On the molecular level, actin-myosin interactions are controlled by, among others, two isoforms of the non-muscle motor protein myosin II, myosin IIA and myosin IIB. Myosin II is a hexameric molecule consisting of 2 heavy chains and 2 sets of paired light chains, the essential light chain and the regulatory light chain. Distinguished by their unique heavy chains, these myosin II isoforms function to bind and contract F-actin $(10,11)$. The activity of the entire myosin II is mainly regulated via posttranslational phosphorylation of myosin regulatory light chain 9 (Myl9; also known as MLC20, RLC-C, or Mylc2c) by the opposing activities of MLC kinases (MLCKs) and a MLC phosphatase (MYP). While these posttranslational modifications of Myl9 are well-documented events predominantly triggered by the RhoA/ROCK signaling pathway $(12,13)$, transcriptional regulation of $M y l 9$ has only been addressed with regard to tissue-specific gene regulation. Recently, Myl9 expression was shown to depend on myocardin-related transcription factors and SRF and to be necessary for cytoskeletal dynamics and experimental metastasis (14).

Previously, members of the activator protein-1 (AP-1) family of transcription factors have been associated with cellular contractility $(15,16)$, with VSMC migration and proliferation in vitro and during neointima formation (17), and with EC migration and sprouting (18-20). AP-1 mediates gene regulation in response to a plethora of extracellular stimuli including stress-induced signals and consists of homo- or heterodimers of Jun, Fos, and ATF proteins (21-23). Interestingly, some phenotypes of mouse mutants with ablated Junb and cells derived thereof display failures that may be 
A

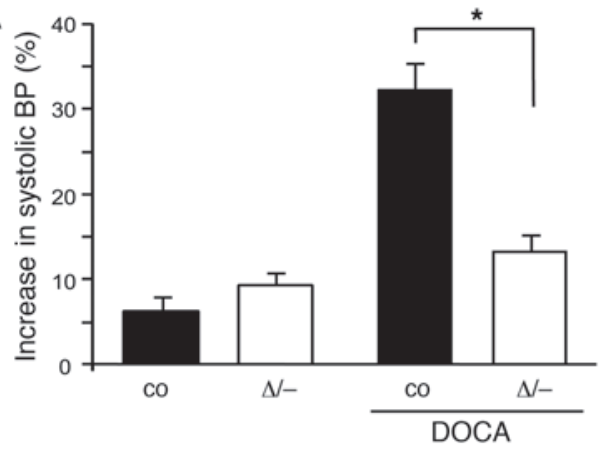

C
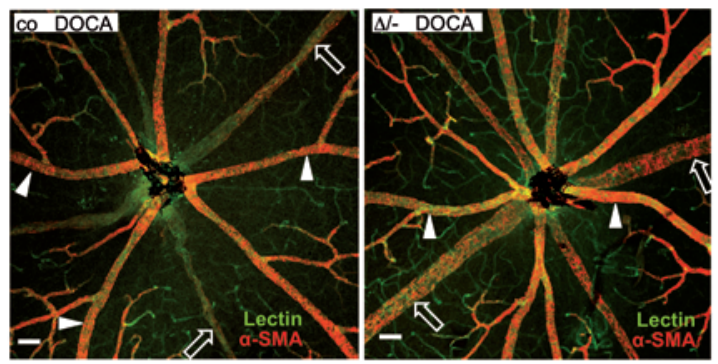

E

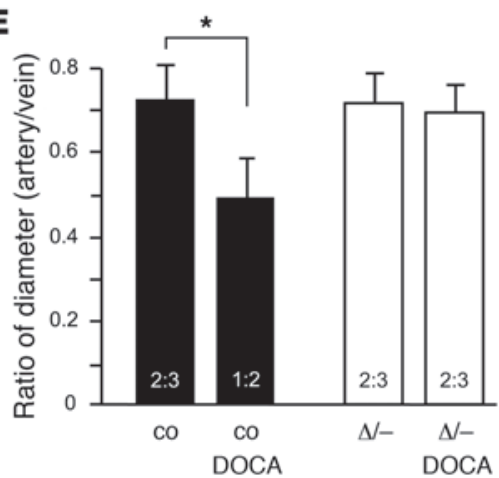

F
B

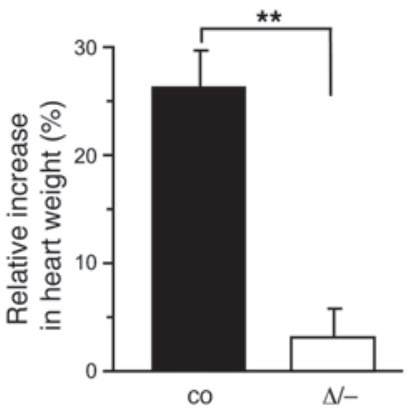

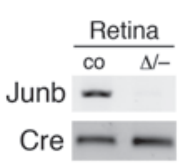

D

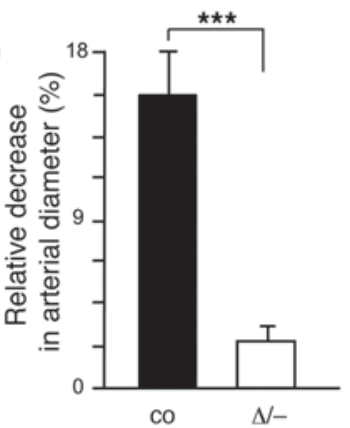

co

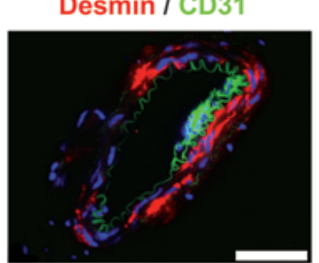

co

DOCA
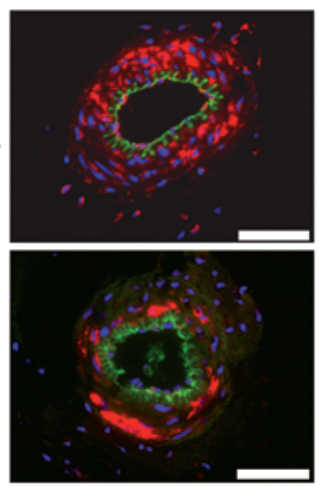

$\Delta /-$

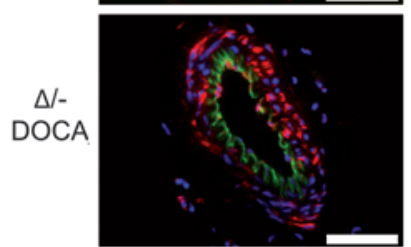

PCNA / CD31
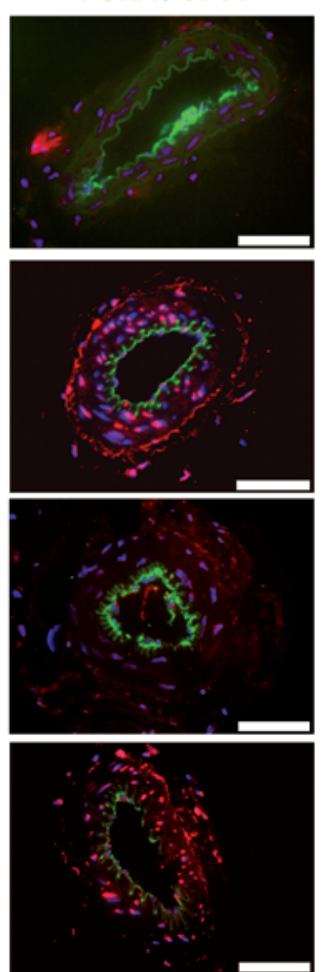

Calponin / CD31
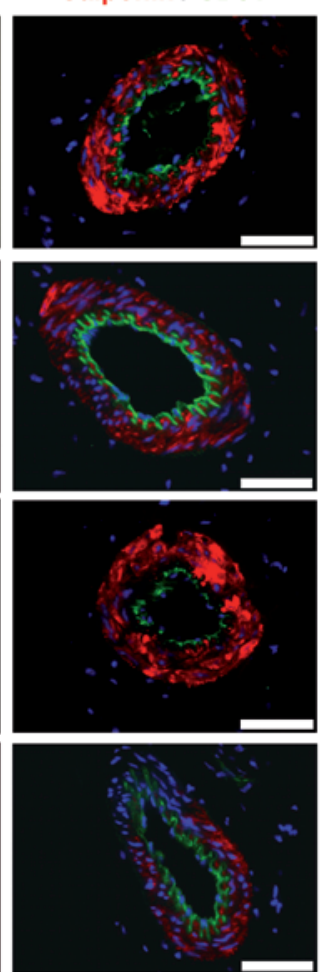

Figure 1

Conditional Junb-deficient mice do not develop hypertension and adaptive responses in the DOCA-salt model. (A and B) Relative increase in systolic blood pressure (A) and in heart weight (B) is depicted for wild-type control (co) and Junb-deficient $(\Delta /-)$ mice subjected to sham operation or DOCA-salt treatment for 21 days. (C) Left: Whole-mount double-staining of retinal vessels with FITC-coupled lectin (green) and $\alpha$-SMA antibody (red). Arrows indicate veins; arrowheads, strongly $\alpha$-SMA-positive arteries. Right: Complete deletion of the Junb locus was verified by PCR with genomic DNA preparations from complete retinas of wild-type and Junb-deficient mice. Col1 $\alpha 2$-iCre (Cre) PCR served as control for equal quality and loading. (D) Relative arteriolar narrowing determined by comparing diameters of arterioles from untreated and DOCA-salt-treated control and $J u n b^{\Delta /-}$ mice within a distance of $250 \mu \mathrm{m}$ from the optical disc. (E) Ratio of artery to vein diameters is plotted for untreated and DOCA-salt-treated control and Junb ${ }^{\Delta-}$ mice. (F) Immunofluorescence costaining of femoral arteries isolated from untreated or DOCA-salt-treated control and Junb ${ }^{\Delta-}$ mice for CD31 and desmin, PCNA, or calponin. Scale bars in C and F: $50 \mu \mathrm{m}$. In each case, one representative staining of sections or of retinas of at least 3 different mice is shown. Data in $\mathbf{A}, \mathbf{B}, \mathbf{D}$, and $\mathbf{E}$ represent mean $\pm \mathrm{SEM}$ ( $n \geq 6$ for each condition). ${ }^{*} P<0.05,{ }^{* *} P<0.01,{ }^{* \star *} P=0.005$. 
associated with actomyosin-mediated cell motility and contraction, such as failure in trophoblast and EC migration $(20,24)$ as well as in fibroblast contractility (16). However, a direct mechanistic link between members of the AP-1 family and actomyosin-based cell motility and contraction has not been provided so far. Complete loss of the AP-1 family member Junb results in embryonic death between embryonic days 8.5 and 10 due to placental failure and defective vascular development (24). A similar phenotype was also seen upon EC-specific Junb ablation in vivo (20). Furthermore, the absolute requirement of Junb for expression of the angiogenic key regulator Vegfa and of Cbfb was shown in vitro and in vivo $(19,20)$.

Due to the fundamental role of Junb in vascular processes, we applied the volume-dependent deoxycorticosterone acetate-salt (DOCA-salt) hypertension model to conditional Junb knockout mice to investigate the impact of Junb loss on VSMC contractility and the maintenance of vascular tone in vivo.

\section{Results}

Junb deficiency results in reduced arterial contractile capacity in vivo and in vitro. Based on our previous findings that Junb-deficient ECs have reduced lateral migration capability (20) and that mouse embryonic fibroblasts (MEFs) lacking Junb expression show a decreased contraction of collagen gels (16), we investigated the contractile behavior of Junb-deficient blood vessels and cells in vivo. To this end, DOCA-salt hypertension was induced in mice with conditionally ablated Junb (Junb $\left.b^{\Delta /-} \mathrm{Col} 1 \alpha 2-\mathrm{iCre}^{+}\right)(16)$, which were generated upon crossing Junb floxed mice to heterozygous Col1 $\alpha 2$-iCre ${ }^{+}$ mice. As controls, corresponding Col1 $\alpha 2$-iCre $\mathrm{Cl}^{+}$littermates were used $\left(J u n b^{\Delta /+} \mathrm{Col} 1 \alpha 2-\mathrm{iCr} \mathrm{C}^{+}\right)$. Jun $b^{\Delta /-}$ mice display shaggy fur as well as reduced body size and body weight (approximately 10\% less than control mice), reminiscent of the phenotype of animals displaying strongly reduced Junb expression in bone $(16,25)$. Changes in arterial blood pressure were monitored over time following DOCA-salt administration. While control animals developed a significant, $33 \%$ increase in systolic blood pressure upon a 21-day DOCA-salt application, the blood pressure of Junb-deficient mice was insignificantly increased, by $16 \%$ (Figure 1A). Untreated controls displayed minor increases of $12 \%$ and $8 \%$, respectively (Figure 1A). Analysis of cardiac hypertrophy, an adaptive response to a pressure-dependent increase in mechanical load of cardiomyocytes $(26,27)$, revealed a $25 \%$ rise in heart weight for control animals, while $J u n b^{\Delta /-}$ hearts displayed no significant weight change upon DOCA-salt application (Figure 1B).

Generalized narrowing of arterioles in the retina has long been recognized as an early feature of hypertension $(28,29)$. In normal healthy blood vessels of the retina, the difference in size between arteries and veins typically is $2: 3$, while myogenic constriction of retinal arteries in response to increased blood pressure results in a reduction of this ratio to less than 1:2 (30). Thus, determining relative changes in the size of small arterial blood vessels of the retina provides an additional means to monitor the in vivo response of this type of blood vessel to DOCA-salt-induced hypertension. Consequently, retinas were isolated from DOCA-salt-treated control and $J u n b^{\Delta /-}$ mice, and diameters of arteries and veins were analyzed by confocal microscopy. Complete deletion of the Junb locus by the activity of Cre recombinase in the retinal compartment was verified by PCR with genomic DNA preparations from complete retinas isolated from control and Junb-deficient mice (Figure 1C). Arteries and veins were distinguished by double-staining of the retinal blood vessels with lectin coupled to FITC and an anti-
$\alpha$-SMA-Cy3 antibody, which resulted in a more pronounced $\alpha$-SMA staining of small arteries and arterioles, and by differences in their vessel branching morphology (Figure 1C). In DOCA-salttreated control and Junb-deficient mice, the diameter of the retinal arterioles was reduced by $16 \%$ and $3 \%$, respectively, as compared with untreated animals (Figure 1D). While DOCA-salt-induced hypertension caused significant changes in the artery/vein ratio from $2: 3$ to $1: 2$ in the retina of control mice, no such arterial contraction was observed in retinal blood vessels of Junb-deficient mice (Figure 1E). Thus, loss of Junb significantly halted secondary syndromes related to DOCA-salt-induced hypertension, such as cardiac hypertrophy and retinal arteriolar constriction.

We further analyzed on a molecular level the susceptibility and responsiveness of blood vessels toward DOCA-salt treatment. For this purpose, femoral arteries were subjected to immunofluorescence staining for distinct marker proteins. Beforehand, deletion of the Junb locus was verified by PCR with genomic DNA preparations from mesenteric arteries isolated from control and Junb-deficient mice (Supplemental Figure 1A; supplemental material available online with this article; doi:10.1172/ JCI41749DS1). Importantly, no morphological or structural differences per se between control and Junb-deficient arteries were detected, as demonstrated by similar expression of desmin (Figure 1F and Supplemental Table 1). Staining for the proliferation marker proliferating cell nuclear antigen (PCNA) confirmed that Junb-deficient as well as control arteries responded to the DOCAsalt-induced hypertension with a profound 2- to 3-fold increase in proliferative activity (Figure 1F and Supplemental Table 1). Release of actin-bound calponin, as verified by more than $50 \%$ loss of calponin staining intensity in DOCA-salt-treated arteries, substantiated an active vascular remodeling process that was not affected by the loss of Junb (Figure 1F and Supplemental Table 1).

To test our hypothesis of an impaired myogenic constriction of Junb-deficient arterioles, we determined the contractile capacity of isolated small arteries ex vivo. Segments of second-order branches of mesenteric arteries were subjected to increasing levels of intraluminal pressure and hence were passively distended. Typically, arteries and in particular arterioles limit such a pressure-dependent distention through active constriction. Junb-deficient mesenteric artery segments were more easily distended upon increasing intraluminal pressure than those derived from control animals (Figure 2A), highlighting a defective contractile capacity.

Using the same setup, mesenteric arteries were subjected to continuous perfusion at a flow of approximately $0.07 \mathrm{ml} / \mathrm{min}$, followed by treatment with the vasoconstrictive agent norepinephrine. Only stimulation of the arterial segments from control mice with norepinephrine resulted in a robust concentration-dependent decrease in diameter (Figure 2B). Junb-deficient arterial segments reacted with a much weaker contraction at all concentrations tested (Figure 2B, left). Administration of acetylcholine (100 $\mu \mathrm{mol} / \mathrm{l}) \mathrm{sub}-$ sequent to the highest concentration of norepinephrine resulted in a comparable near-maximum arterial relaxation, showing that the analyzed segments were still endothelium-intact (data not shown). Additionally, we analyzed the contractile capacity of denuded arterial segments by exposing them to increasing concentrations of norepinephrine (Figure 2B, right). Constriction of de-endothelialized arteries requires stimulation by higher concentrations of norepinephrine, as the vessel wall has been traumatized by the denuding procedure, limiting its contractile capacity. Removal of ECs was validated (a) functionally, by failure of acetylcholine- 


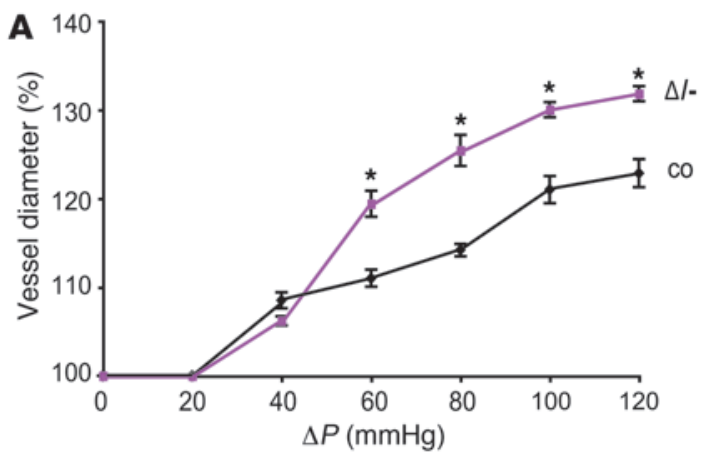

B
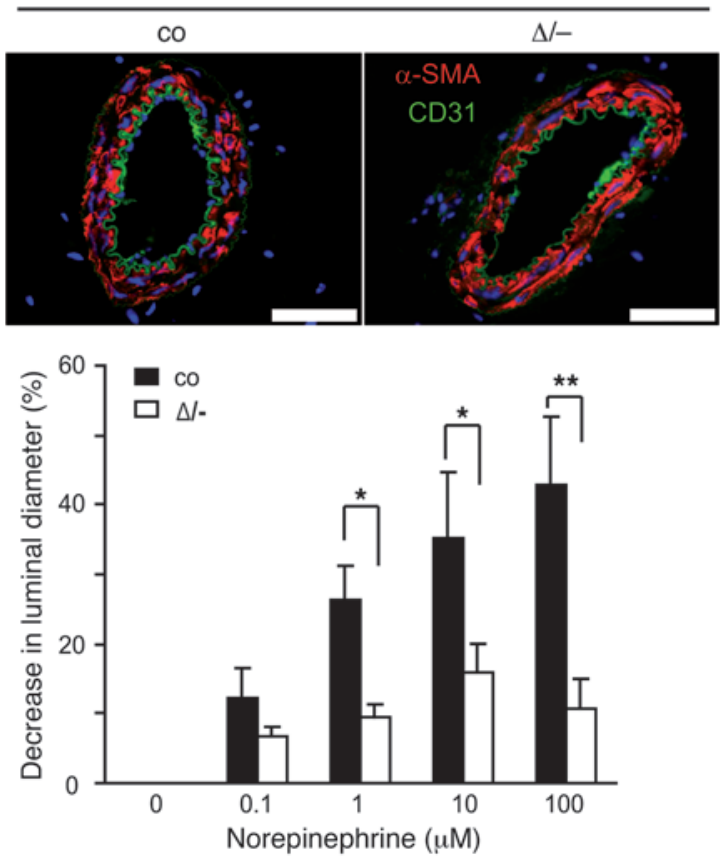

De-endothelialized vessel
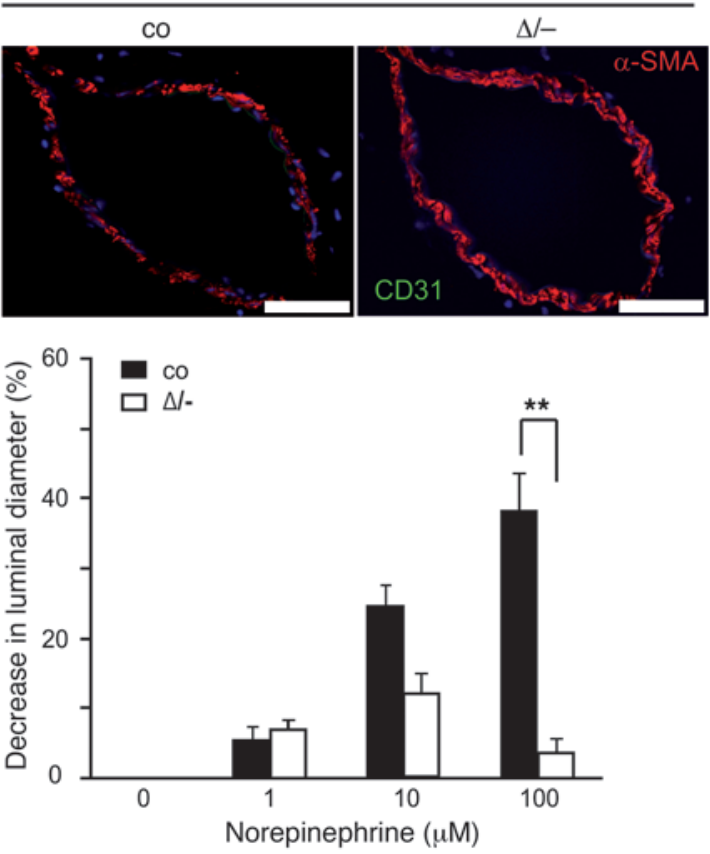

Figure 2

Contraction failure of second-order mesenteric artery branches derived from Junb-deficient mice. (A) Relative increase in diameter of mesenteric artery segments from control and $J u n b^{\nu-}$ mice challenged with increasing intraluminal pressure. Diameter of unchallenged arteries was set to $100 \% . \Delta P$, pressure difference at which segments were perfused. Results were obtained from 6 independent segments from 3 individual mice of each genotype. (B) Concentration-dependent decrease in luminal arterial diameter of mesenteric artery branches derived from control and $J u n b^{\Delta-}$ mice upon treatment with the vasoconstrictive agent norepinephrine is shown. Mesenteric artery branches were denuded by perfusion with an incompatible culture medium (right) or left intact (left) prior to norepinephrine exposure. Vessel integrity or EC removal was confirmed by immunofluorescence costaining for CD31 and $\alpha$-SMA. Nuclei were counterstained with Hoechst 33342 (blue). Scale bars: $200 \mu \mathrm{m}$. In each case, one representative staining of sections of at least 3 different mice is shown. Data are presented as mean $\pm \mathrm{SEM}(n=6)$. ${ }^{* \star} P<0.01,{ }^{\star} P<0.05$.

induced dilation of norepinephrine-preconstricted arteries (data not shown), and (b) by staining for CD31 and $\alpha$-SMA (Figure 2B). In line with the aforementioned results, denuded Junb-deficient mesenteric artery segments displayed a failure in contractile capacity similar to that of their endothelium-intact counterparts.

Junb controls stretch-induced stress fiber formation and motility. VSMCs are key effectors in the wall of blood vessels, determining their tone, resistance, and perfusion through their contractile capacity $(1,31)$. As stress fiber formation provides a mechanism for both structural and biochemical adaptation of VSMCs to mechanical load (6), we mimicked the effect of pressure-induced deformation by subjecting cultured VSMCs to cyclic stretch followed by analysis of subsequent stress fiber formation. While control VSMCs exhib- ited prominent stress fiber formation 3 hours after cyclic stretch, this was absent in Junb-deficient VSMCs (Figure 3A). Moreover, in resting Junb-deficient VSMCs, no stress fibers were detected at all in comparison to a marginal staining in control cells (Figure 3A). Stress fibers are rod-shaped structures made by the actomyosin-based contractile systems in several cell types, including fibroblasts. Concomitantly, we analyzed stress fiber formation in MEFs lacking Junb. In wild-type fibroblasts, prominent F-actin-positive bundles could be readily detected, while stress fibers were nearly absent in Junb-deficient MEFs and, in addition, were much thinner compared with those of control MEFs (Figure 3A).

Formation of stress fibers is essential for cell motility. Specifically, migrating cells retract their tails by contraction of stress fibers 
A
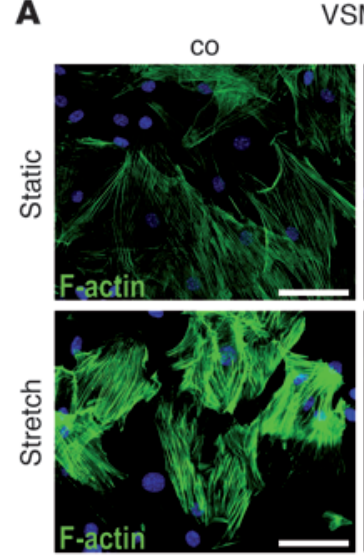

VSMCs

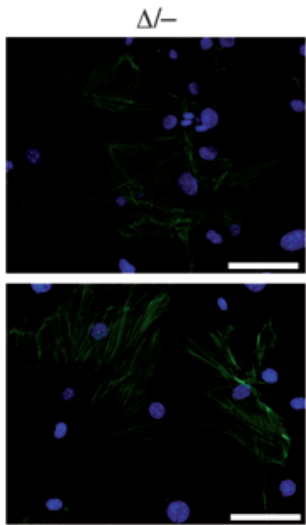

MEFs

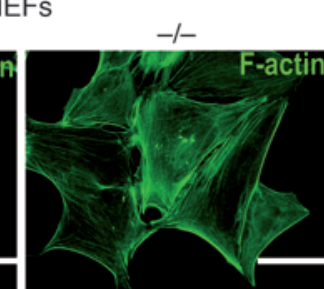

B

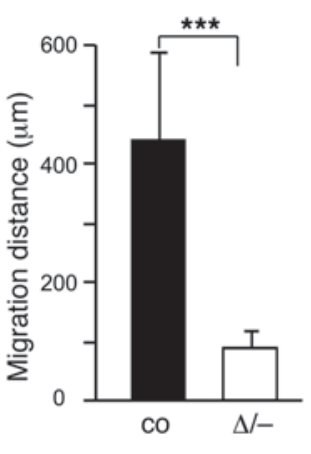

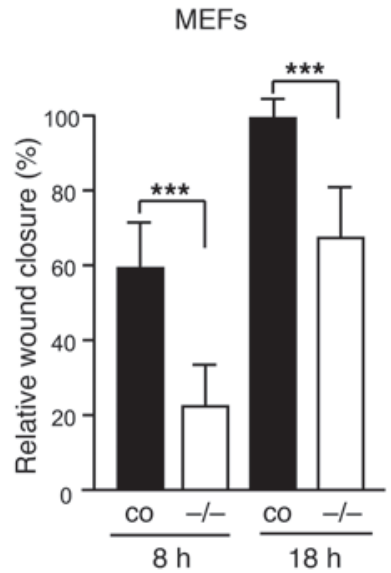
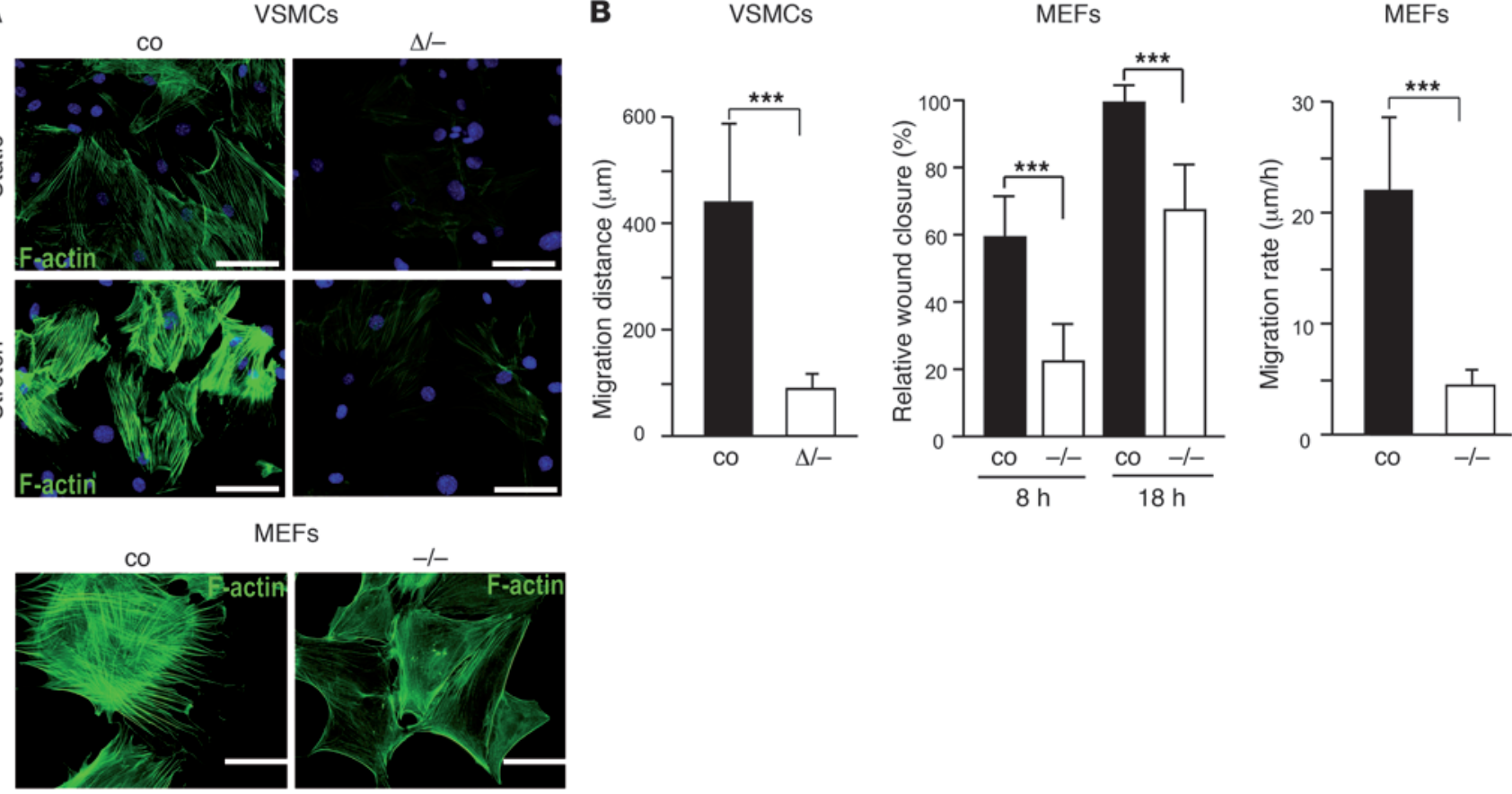

Figure 3

Stress fiber formation and migration of VSMCs and MEFs is Junb-dependent. (A) Top: Stress fiber formation in control and Junb ${ }^{\Delta /-}$ VSMCs was monitored by immunofluorescence staining with phalloidin-Alexa Fluor 488 (green) at 0 (Static) and 3 hours after cyclic stretch application (Stretch). Nuclei were counterstained with Hoechst 33342 (blue). Bottom: Stress fibers were stained in control and Junb ${ }^{-/-}$MEFs with phalloidin-Alexa Fluor 488 (green). Scale bars: $10 \mu \mathrm{m}$. One representative staining of at least 3 independent cell preparations is shown ( $n \geq 3$ ). (B) Left and middle panels: Relative wound closure of control and Junb-deficient VSMCs $(\Delta /-)$ and MEFs $(-/-)$ within 4 days (VSMCs) and 8 hours or 18 hours (MEFs) after wounding. Right: Average length of tracks (in $\mu \mathrm{m} / \mathrm{h}$ ) from 10 wild-type and 10 Junb $\mathrm{J}^{-/}$MEFs each over a period of 8 hours. Error bars represent SD (left and middle panels) and SEM (right panel) values (in each case, $n \geq 3$ ). ${ }^{* * *} P<0.005$.

(12). As we have shown previously that Junb-deficient ECs have a reduced lateral migration capacity (20), we now analyzed the migratory capacity of Junb-deficient VSMCs and MEFs. Confluent monolayers were scratch-wounded (wound of 1-mm width), and migration was determined by monitoring wound closure by the cells over time. While control VSMCs achieved a distance of about $447 \pm 144 \mu \mathrm{m}$, Junb-deficient VSMCs migrated a 4.5-fold-shorter distance (Figure 3B). Consistent with this finding, Junb-deficient MEFs showed a clearly retarded gap closure in a scratch-wounding assay (Figure 3B). While wild-type cells closed the gap almost completely within 18 hours after wounding, cells lacking Junb covered only two-thirds of the wound area. This difference was even more evident at the earlier time point of 8 hours after wounding (Figure 3B). To further analyze cellular motility on the single cell level, we applied video microscopy to track cell movements. In this assay, wild-type cells migrated approximately 4-fold faster than Junbdeficient MEFs (Figure 3B).

$J u n b^{\Delta /-}$ arteries and cells express reduced levels of Myl9. Actin polymerization, reorganization, assembly of stress fibers, as well as retraction of the cell tail during migration require phosphorylation of Myl9 (12, 13, 32-34). Therefore, the phosphorylation status and overall protein levels of Myl9 were analyzed. The amount of phosphorylated Myl9 was strongly reduced in both native tissue and cultured cells lacking Junb, including VSMCs of retinal and mesenteric arteries, MEFs, as well as isolated primary VSMCs (Figure 4A and Supplemental Table 1) and ECs (Supplemen- tal Figure 2A). By contrast, the corresponding control tissue or cells revealed robust phosphorylation of Myl9 (Figure 4A and Supplemental Figure 2A). Analysis of femoral arteries isolated from DOCA-salt-treated mice revealed that hypertension and hence volume-induced stretching of vascular cells, in particular of VSMCs, resulted in a slight increase in Myl9 protein (Supplemental Figure 1B and Supplemental Table 1) and elevated levels of phosphorylated Myl9 (Supplemental Figure 1C and Supplemental Table 1). In accordance with the lack of hypertension development and the observed decrease in arterial contractile capacity (Figure $1 \mathrm{~A}$ and Figure 2A), Junb-deficient arteries displayed only marginal levels of Myl9 and phospho-Myl9 protein that were only slightly changed upon DOCA-salt treatment (Supplemental Figure 1, B and C, and Supplemental Table 1).

Myl9 is a Junb target gene. Remarkably, severely reduced abundance of total Myl9 protein was observed in Junb-deficient VSMCs, MEFs (Figure 4B), and ECs (Supplemental Figure 2A). Moreover, culture of MEFs on different extracellular matrix components did not increase Myl9 protein levels in Junb-deficient cells (Supplemental Figure 2C). Taken together, these findings supported the idea that Myl9 may be a direct Junb target gene. Indeed, semiquantitative RTPCR analysis revealed a reduced number of Myl 9 transcripts in Junbdeficient VSMCs, MEFs (Figure 4B), and ECs (Supplemental Figure 2A). A 6-fold-higher Myl9 expression was found by quantitative RTPCR (qRT-PCR) in control cells when compared with Junb-deficient VSMCs (Figure 4C). Additionally, reexpression of Junb upon retro- 
A
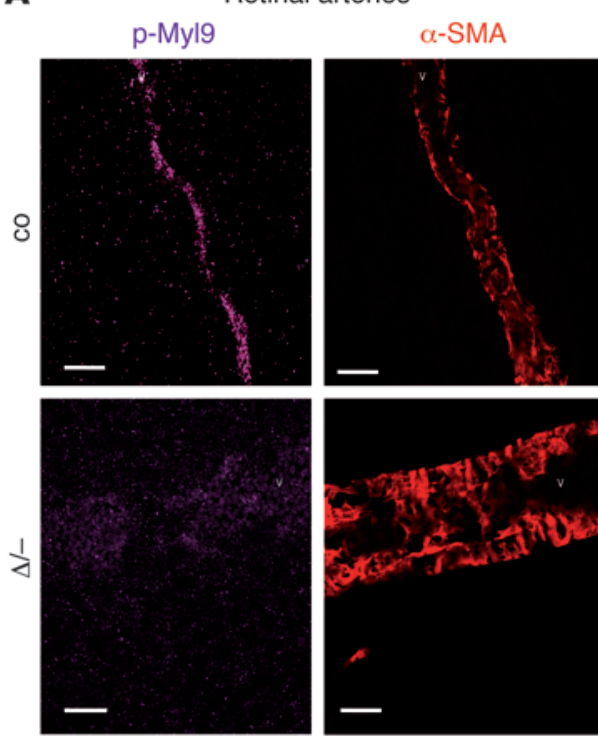

VSMCs
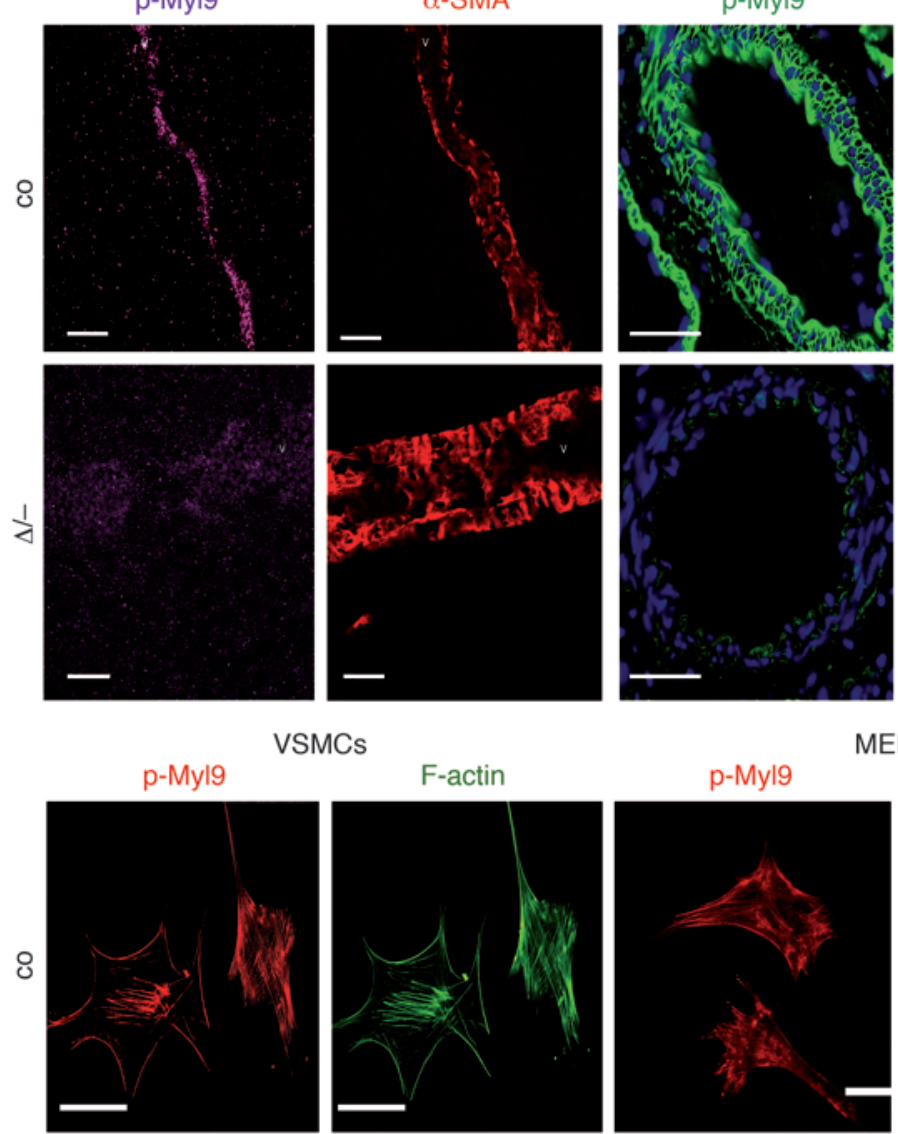

Mesenteric arteries
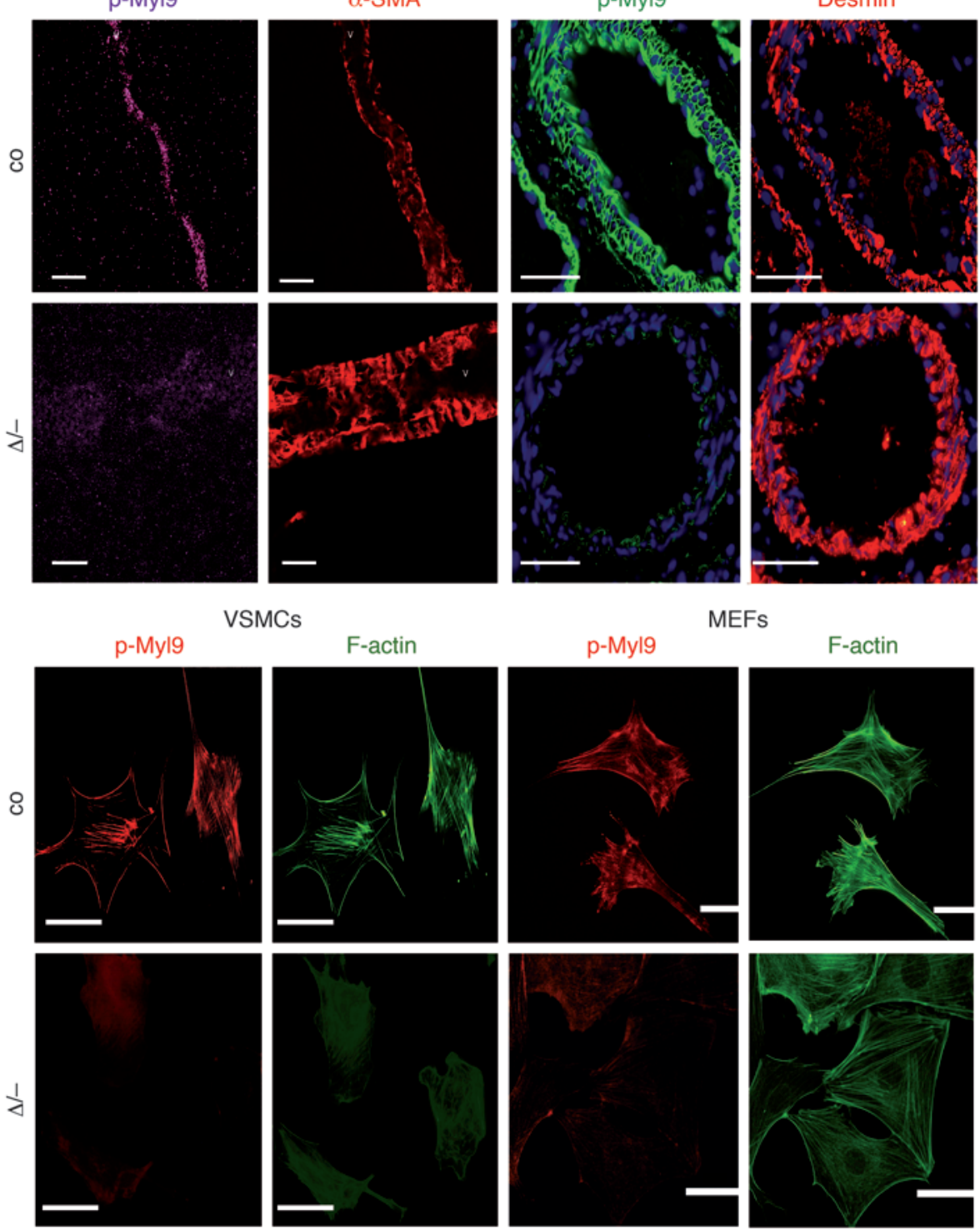

MEFs
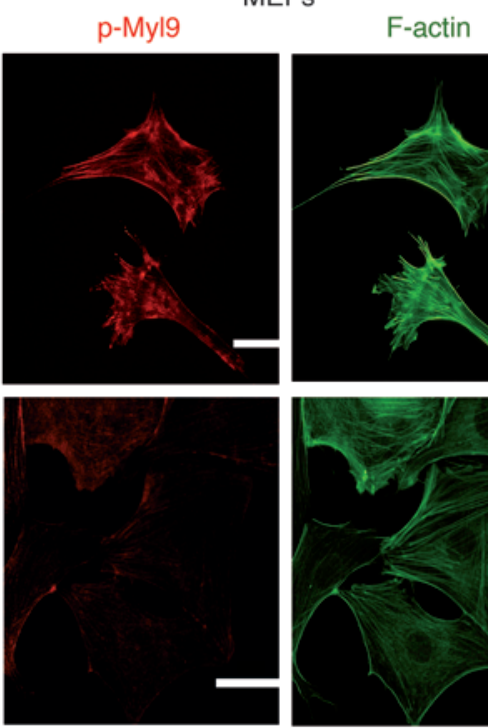

B

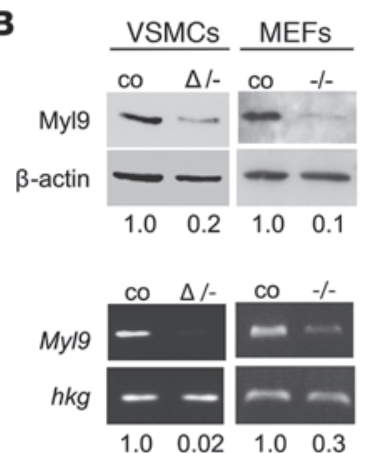

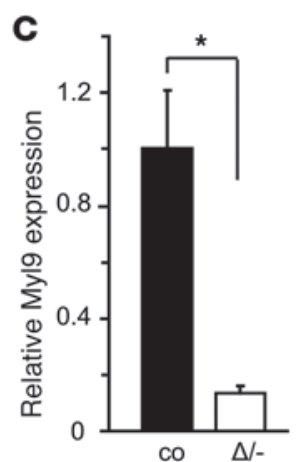

Figure 4

Jun $b^{\Delta /-}$ arteries and cells show severely diminished levels of Myl9 protein and mRNA. (A) Levels of phosphorylated Myl9 were assessed by immunofluorescence staining with a specific antibody recognizing phospho-Ser19 of Myl9 (p-Myl9) in control and Junb ${ }^{\Delta /-}$ retina preparations, mesenteric artery branches, primary VSMCs, and MEFs. Retinal artery wholemount sections were costained with an $\alpha$-SMA (red) antibody for VSMC coverage. Mesenteric arteries were costained with anti-desmin (red; nuclei were counterstained with Hoechst 33342 [blue]); VSMCs and MEFs were costained with phalloidin-Alexa Fluor 488 (green) to visualize stress fibers. Scale bars: $20 \mu \mathrm{m}$ (retinal arteries), $200 \mu \mathrm{m}$ (mesenteric arteries), and $10 \mu \mathrm{m}$ (VSMCs, MEFs). In each case, one representative staining of at least 3 different sections or cell preparations is shown $(n \geq 3)$. (B) Top: Immunoblot analysis reveals diminished Myl9 protein levels in Junb ${ }^{\Delta /-}$ VSMCs and Junb ${ }^{-/-}$MEFs. Bottom: Semiquantitative RT-PCR analysis confirms reduced expression of $M y / 9$ in Junb ${ }^{\Delta /-}$ VSMCs and Junb ${ }^{-/-}$MEFs at the mRNA level. Expression analyses of housekeeping genes $(h k g)$ served as control for equal quality and loading. Results of densitometric quantification of blots are indicated under each lane. One representative result of 3 independent experiments is shown $(n=3)$. (C) qRT-PCR analysis confirmed the results of $\mathbf{B}$, showing a 6-fold-reduced expression level of Myl9 in $J_{u n b^{\Delta-}}$ VSMCs. Error bars represent SD values $(n=3)$. ${ }^{*} P<0.05$. viral transduction of $J u n b^{\Delta /-}$ VSMCs restored Myl9 protein levels, as shown by immunoblot analysis (Supplemental Figure 2B).

To demonstrate that $M y l 9$ is a direct Junb target gene, we analyzed Myl9 promoter occupation in MEFs and endothelioma cells (END cells) (20) by ChIP analysis (Figure 5, A and B). For this purpose, primer sets were generated to amplify distinct regions of the Myl9 promoter (Figure 5A). Two regions amplified by primer pairs P2 and P3 contain putative Junb binding sites, such as TPA response elements (TREs) or cAMP response elements (CREs), while region $\mathrm{P} 1$ recognized by primer set $\mathrm{P} 1$ and located 3,000 

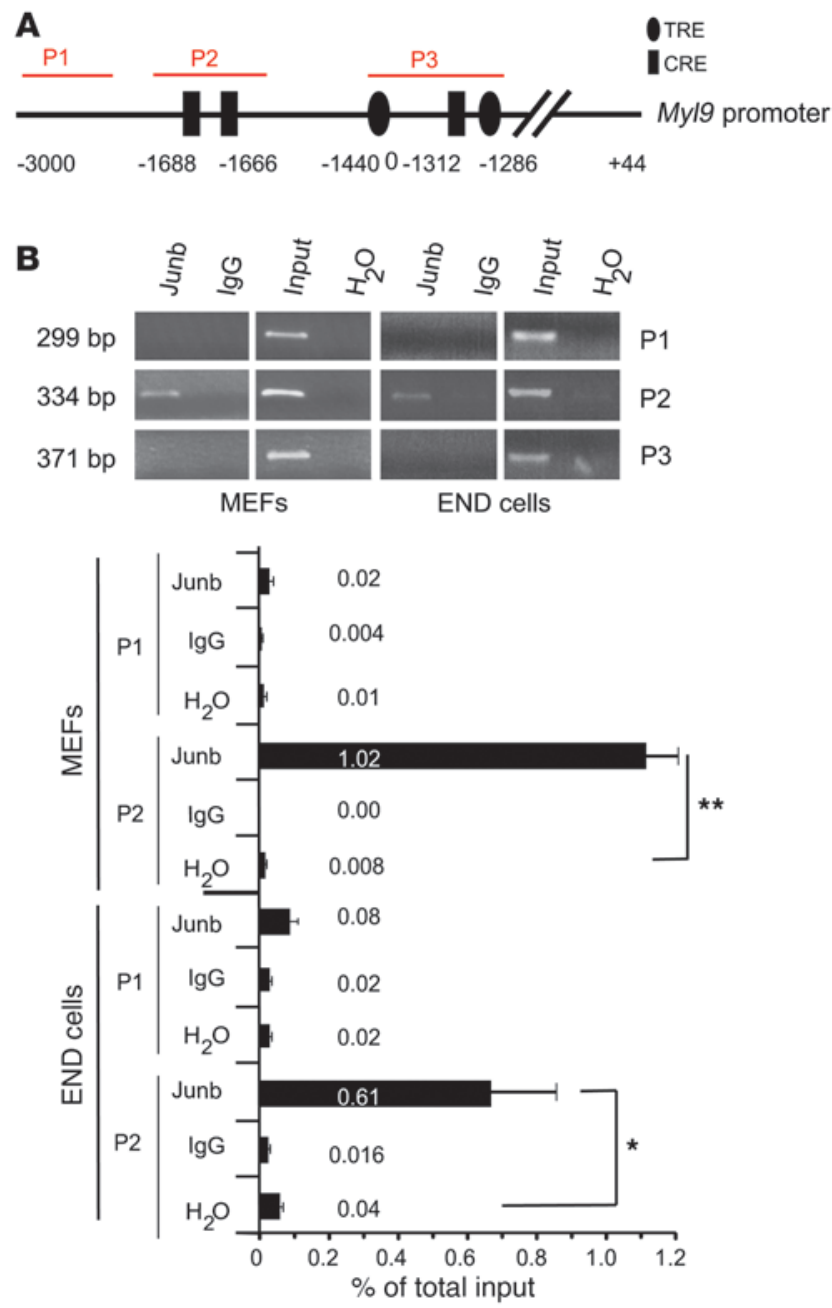

base pairs upstream of the transcriptional start site of Myl9 lacked Junb binding sites and thus served as negative control. ChIP analysis with a specific Junb antibody revealed binding of Junb to the region amplified by primer pair P2 (Figure 5B). This region contains 2 CREs in close proximity at positions $-1,688$ and $-1,666$ upstream of the transcriptional start site. In contrast, the Junb antibody failed to immunoprecipitate the irrelevant control region P1 and the region P3 harboring 2 classical TREs and 1 CRE (Figure 5B). Quantitative PCR (qPCR) analysis revealed that the Junb antibody precipitated $1 \%$ and $0.61 \%$ of total input chromatin from MEFs and END cells, respectively, for the target region P2. qPCR with control primers flanking an irrelevant region (P1) or on IgGprecipitated chromatin did not yield significant amounts of PCR product (Figure 5B). Hence, Junb binds to the 2 CREs at positions $-1,688$ (TGACATCA) and -1,666 (TGACATCA), suggesting that Myl9 is a direct Junb target gene.

Reexpression of Myl9 in Junb-deficient cells restores cellular motility and contractility. To further validate our data and to assess whether exclusively diminished Myl9 expression due to lack of Junb is causative for the aforementioned phenotypes, we reexpressed either Junb or Myl9 in Junb-deficient MEFs. Successful protein reexpression was confirmed by immunoblot analysis (Figure 6, A and B). Junb-transduced cells showed a clear reexpression of Myl9 as compared with Junb-deficient MEFs transduced with empty vector (Figure 6A).

\section{Figure 5}

Myl9 is a Junb target gene. (A) Scheme representing the Myl9 promoter region with putative Junb binding sites and regions (P1-P3) targeted during ChIP analyses. (B) ChIP analysis on the Myl9 promoter was performed for wild-type MEFs and END cells using an antibody specific for Junb or rabbit IgG as a negative control as indicated. Top: Representative ethidium bromide-stained agarose gel. Bottom: qPCR analyses using primer pairs $\mathrm{P} 2$ and $\mathrm{P} 1$ amplifying the region spanning the 2 CRE sites or an irrelevant region, respectively. Relative amount of chromatin precipitated by the Junb antibody and the control rabbit IgG are given as percentage of input material set to $100 \%$. Results represent the mean \pm SD of 3 independent experiments, each carried out in triplicate. ${ }^{*} P<0.05,{ }^{* *} P<0.01$.

Notably, either sole Junb or sole Myl9 reexpression restored stress fiber formation, in contrast to cells transduced with empty vector (Figure 6, C and D). Moreover, the migratory behavior of Junb- as well as Myl9-rescued cells was virtually identical to that of control cells, as demonstrated by a completely reconstituted wound closure capability (Figure 6, E and F). Formation of stress fibers as well as migration capability of wild-type MEFs was not influenced by overexpression of Junb or Myl9 (Supplemental Figure 3, A-C). Most importantly, reintroduction of Myl9 in a Junb-deficient background restored levels of both Myl9 (Figure 6B) and phosphorylated Myl9 (Figure 6D). Thus, a potential defect in the upstream signaling cascade regulating stress fiber assembly can be excluded.

In order to directly determine the ability of Junb-and Myl9-rescued cells to reorganize and contract extracellular matrix, we performed a collagen gel contraction assay previously used to demonstrate a contraction failure of Junb-deficient primary skin fibroblasts (16). In line with our previous findings, Junb-deficient MEFs showed a severe impairment in contracting collagen lattices. While wild-type cells displayed a contraction capacity of $58 \%$, Junb $b^{-/}$MEFs or empty vector-transduced $\mathrm{Junb}^{-/-}$cells exhibited a contraction capacity of only $5.8 \%$ or $1.8 \%$, respectively (Figure $6 \mathrm{G}$ ). By contrast, reexpression of either Junb or Myl9 resulted in a major improvement of contraction capacities, i.e., $49.9 \%$ and $38.5 \%$, respectively (Figure 6G). Overexpression of either Junb or Myl9 in wild-type cells also had no influence on the contractile capacity of fibroblasts (Supplemental Figure 3D). As defects in cellular motility and contraction capacity of Junb-deficient MEFs could be reestablished upon unique Myl9 reexpression (Figure 6, F and G), impaired stress fiber formation and cell contractility and motility were predominantly caused by the sole loss of Junb-dependent Myl9 protein expression.

\section{Discussion}

Hypertension is a critical risk factor for cardiovascular diseases and is usually associated with other health threats, such as aging, adiposity, insulin resistance, diabetes, and dyslipidemia $(3,35,36)$. Treating hypertension has been shown to decrease the risk of developing heart failure. Today, the most challenging tasks deal with the accurate diagnosis and selection of the optimal treatment regimen $(35,37)$. A broad understanding of the underlying vascular biology and molecular mechanisms is a prerequisite to efficiently reducing cardiovascular morbidity and mortality. Here, we have applied the well-known volume-dependent DOCA-salt model of hypertension to genetically modified mice lacking Junb and were able to demonstrate that loss of this transcriptional regulator prevents mice from developing hypertension and associated adaptive responses such as cardiac hypertrophy and retinal artery constric- 

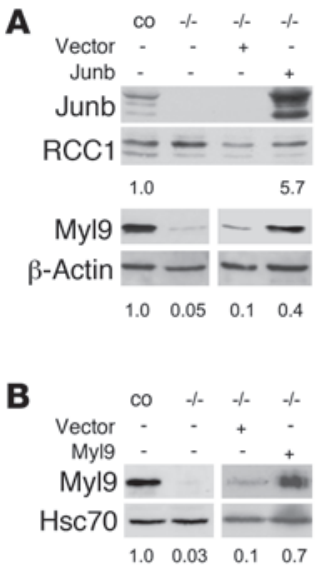

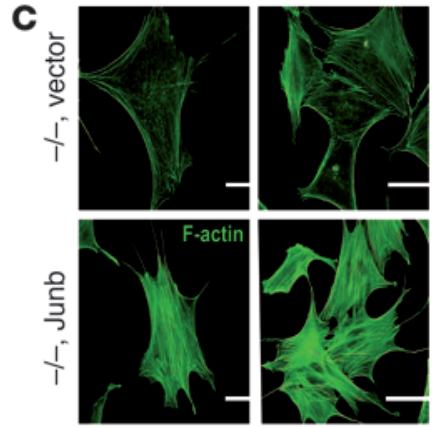

D

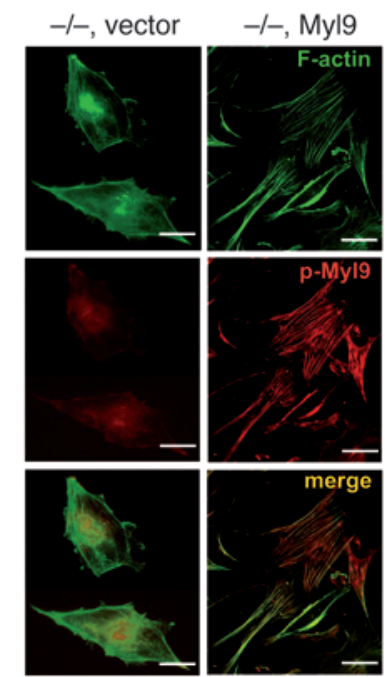

Figure 6

Reexpression of Junb or Myl9 in Junb ${ }^{-/-}$cells restores stress fiber formation, cellular motility, and contraction capability. (A and B) Reexpression of Junb or Myl9 in Junb-/- MEFs restores Myl9 expression, as confirmed by immunoblot analysis of nuclear and total extracts from wild-type MEFs (co) or Junb-l- MEFs that were left untreated or retrovirally transduced. $\mathrm{RCC} 1, \mathrm{Hsc} 70$, and $\beta$-actin served as control for equal quality and loading. Results of densitometric quantification of blots are shown. (C and D) Immunofluorescence staining for F-actin and phosphorylated Myl9 (p-Myl9) on Junb-/- MEFs transduced with an empty vector or Junb- or Myl9-encoding retroviral expression vector. Scale bars: $10 \mu \mathrm{m}$. In $\mathbf{C}$ and $\mathbf{D}$, one representative staining of at least 3 independent cell preparations is shown $(n \geq 3)$. ( $\mathbf{E}$ and $\mathbf{F})$ Relative wound closure of control and $J_{u n b^{-1}}$ MEFs and of the same cells transduced with empty vector or Junb- or Myl9-containing vector subjected to scratch wounding. Values were calculated from images of the wound area taken at 0,8 , and 18 hours after wounding. ${ }^{* * *} P<0.005$. (G) Relative collagen gel contraction by wild-type or Junb $b^{-/-}$MEFs transduced with empty vector or Junb- or Myl9-encoding retroviral expression vector. Gel contraction capability was determined 96 hours after seeding as percentage of the initial gel surface area that was set to $100 \%$. Results represent the mean \pm SD of 3 independent experiments $(n=3)$, each performed in triplicate $(\mathbf{E}$ and $\mathbf{G})$. Bonferroni multiple comparisons test was applied for statistical analysis. ${ }^{\star \star \star} P<0.001$.

tion. Nevertheless, DOCA-salt administration induced structural remodeling of Junb-deficient arteries, as confirmed by an increase in proliferating VSMCs and calponin release from actin filaments, which was comparable to structural changes observed in control vessels (Figure $1 \mathrm{~F}$ ). Yet these arteries did not contract properly in response to an increase in blood volume and hence hydrostatic pressure. Most likely, this is caused by a failure in actomyosinmediated vessel contractility. We could identify as a responsible molecular mechanism a decreased capacity for stress fiber formation in vascular cells, predominantly in VSMCs, and subsequently a reduced cellular contractility caused by the blunted expression
E

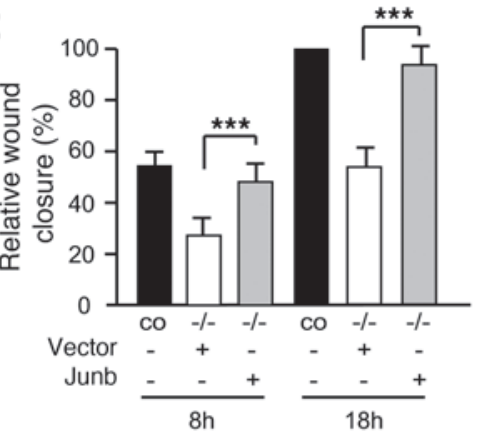

$\mathbf{F}$

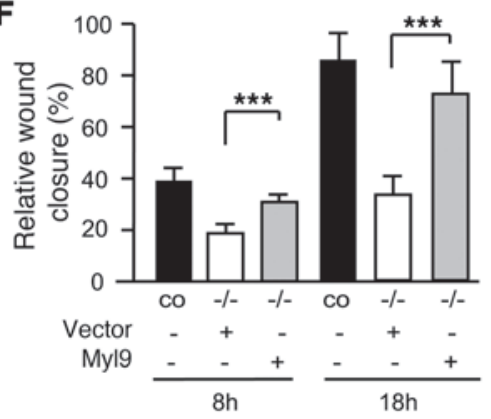

G

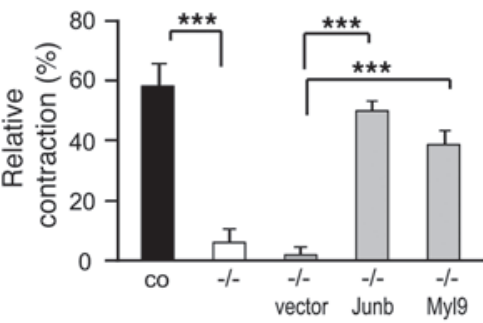

of the novel Junb target Myl9 (Figure 7). Our findings that animals with conditionally ablated Junb were unable to efficiently counteract the increased volume/hydrostatic pressure-dependent distention by active constriction and, as a consequence, did not develop hypertension link Junb directly to disease patterns such as hypertension or vasospasm.

Until now, strong evidence has accumulated that actomyosin-based cell contractility and motility are mainly controlled by phosphorylation of Myl9. This Myl9 phosphorylation on Ser19 causing the contraction of the actin network by myosin II is regulated by various MLC kinases and myosin phosphatase (MYP) (Figure 7). While MLCK, ROCK, ZIP kinase, and citron kinase directly phosphorylate Ser19 of Myl9, maintenance of phospho-Myl9 is achieved via ROCK-mediated phosphorylation and hence inactivation of MYP $(12,13)$. The activities of these various protein kinases are controlled via distinct signaling pathways involving FAK, Rho/ROCK, integrin-linked kinase (ILK), and protein kinase C. Initial triggers for these signaling pathways are $\mathrm{Ca}^{2+}$ sensitization and extracellular stimuli. Indeed, gene targeting and inhibitor approaches have underscored the implication of the RhoA/ ROCK pathway in the pathogenesis of hypertension (38, 39 ) and the requirement of ILK for the formation of a unitary layer of aligned VSMCs around arterioles and the regulation of blood vessel constriction (40).

Our data show for the first time to our knowledge that a stress-induced transcriptional regulator, namely Junb, is causally involved in the regulation of blood vessel contraction capacity and, moreover, in the basic cellular process of actomyosin-based cellular contractility and motility. Junb directly governs the activity of the non-muscle motor protein myosin II by controlling the abundance of Myl9. ChIP analyses demonstrated binding of Junb to 2 CRE sites present in the promoter of the Myl9 gene. In fact, we could identify impaired Myl9 transcription as a rate-limiting event causally linked to the observed phenotypes, namely, the impaired contractile capacity of Junb-deficient arteries in vivo 


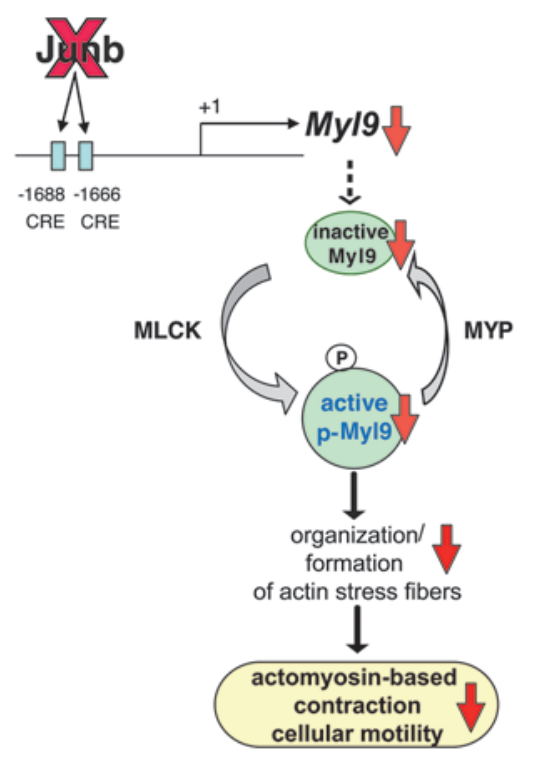

and in vitro, the impaired stress fiber formation and motility of VSMCs, MEFs, and ECs, as well as the defective contractility of MEFs derived from Junb-deficient mice.

In support of these findings, we were able to restore cell motility and contractility upon sole reexpression of Myl9 in Junb-deficient cells. Importantly, mere transcriptional induction of Myl9 expression is Junb dependent, while upstream signaling mechanisms leading to the activation of Myl9 are unaffected, as shown by normal expression and phosphorylation levels of FAK (data not shown) and, most importantly, a robust phosphorylation of ectopically expressed Myl9 in the absence of Junb. Therefore, in addition to the current concept of a primarily posttranslational regulation of Myl9 activity via phosphorylation/dephosphorylation $(12,13)$, Junb-mediated regulation via its expression level plays a pivotal role in Myl9 functionality. The transcriptional regulation of Myl9 by myocardin-related transcription factors and serum response factors (14) raises the possibility that these factors may cooperate with Junb in the transcriptional control of Myl9 by an as-yet-unknown mechanism. Junb-dependent Myl9 expression and the control of cellular contractility and motility linked therewith (Figure 7) may also explain some phenotypes reported for Junb-deficient embryos and mice, such as failure in trophoblast migration, fibroblast contractility, and EC sprouting induced angiogenesis (16, 20, 24).

In this context, it is interesting to mention that processes of both endothelin-mediated vasoconstriction and cold-induced cutaneous vasoconstriction of VSMCs apparently converge at the stimulation of AP-1 activity. Endothelin stimulates ERK activation and subsequent AP-1 transcriptional activity (41). Cold-induced vasoconstriction is initiated by increased expression of cutaneous smooth muscle $\alpha_{2 \mathrm{C}}$-adrenoceptors ( $\alpha_{2 \mathrm{C}}$-Ars), which is mediated through a cyclic AMP/Rap1/JNK/AP-1 signaling pathway (42). For the future, it remains to be demonstrated whether induction of vasoconstriction for both events also involves transcriptional induction of $M y l 9$ via Junb. Generation of conditional Junb knockout mice lacking Junb specifically in VSMCs will provide a valuable tool to study the involvement of Junb-dependent Myl9 regulation, especially in the cold-pressure response.

Aberrant regulation of cellular motility and contractility represent critical parameters of tumor cell invasion and metastasis. In this

\section{Figure 7}

Model showing the molecular link between Junb and actomyosin-based cellular contraction and motility. Junb controls abundance of Myl9 transcripts by transactivating the $M y / 9$ promoter via 2 CRE sites. Subsequent phosphorylation of the Myl9 protein by activated MLCK leads to contraction, while dephosphorylation by MYP promotes relaxation. Junb ablation results in diminished levels of $M y / 9$ and consequently of phospho-Myl9, causing a failure in actomyosin-based contraction.

regard, our data may contribute to the understanding of migration, invasion, and metastatic spread of tumor cells. In fact, overexpression of Junb has been reported for several human tumors/tumor models $(43,44)$, while Myl9 expression levels have not yet been addressed in the context of tumor progression and metastasis.

In the present study, we show that Junb is a key regulator of a pivotal basic cellular function, namely of actomyosin-based contractility and motility. In the course of this, our data obtained with a mouse model showing a broad Junb ablation indicate that Junb is a crucial regulator of arterial contractility. We assume that Junb predominantly affects VSCM function, as the failure in contractile capability persisted in denuded Junb-deficient arteries. Transcriptional upregulation of $M y l 9$ may represent an additional means for cells including VSMCs to deal with stress cues. In the future, it will be of major interest to study VSCM-specific mouse models with a Junb ablation or overexpression in order to investigate the impact of Junb on VSMC biology and physiology in more detail. Overexpression of Junb could be implicated in the abnormally increased contractility of VSMCs observed in vascular diseases such as hypertension and vasospasm superimposed on atherosclerosis. Our findings that interference with Junb function hampers Myl9 abundance and thereby actomyosin-mediated cytoskeletal functions is of fundamental importance with regard to therapeutic strategies targeting MAP kinase signaling and AP-1 activity. Refined approaches to modulate or to soften cellular responses to vascular insults may be required for the development of future antihypertensive pharmacotherapy and treatment of cardiovascular disease and cancer progression.

\section{Methods}

Animal experiments. The generation of Junb ${ }^{+/-}(24)$, Junb floxed (25), and collagen type I a2-iCre (16) mice has been described previously. The genetic background of all mouse lines used was C57BL/6 $\left(>\mathrm{F}_{12}\right)$. Col1 $\alpha 2-\mathrm{iCre}^{+}$ mice were crossed to $\mathrm{Junb}^{+/-}$mice, and iCre-positive males were mated to $J u n b^{f l / f l}$ female mice to obtain Junbll/-Col1 $\alpha 2-\mathrm{iCre}^{+}, J u n b^{f l /+} \mathrm{Col} 1 \alpha 2-\mathrm{iCre}^{+}$, $J u n b^{f /-} \mathrm{Col} 1 \alpha 2-\mathrm{iCre}^{-}$, and $J u n b^{f /+} \mathrm{Col} 1 \alpha 2-\mathrm{iCre}^{-}$mice. Genotypes were detected at the expected Mendelian ratio. Due to Cre activity, Jun $b^{f /-} \mathrm{Col} 1 \alpha 2-\mathrm{iCre}^{+}$and $J u n b^{f /+} \mathrm{Col} 1 \alpha 2-\mathrm{CCre}^{+}$genotypes resulted in the $J u n b^{\Delta /-}$ mice used in the study and in $J u n b^{\Delta /+} \mathrm{Col} 1 \alpha 2$-iCre ${ }^{+}$littermates that were used as controls. Genotyping and housing of mice was done as previously described $(16,24,25)$. All procedures performed on animals were approved by the local government authorities (Regierungspräsidium Karlsruhe, AZ 621-2531.31-1/01).

Twenty-one-day-release DOCA pellets containing $50 \mathrm{mg}$ DOCA (Innovative Research of America) were implanted subcutaneously by incision of the right flank in mice at an average weight of 14-18 g. Control animals were sham operated. All animals (DOCA and control groups) received isotonic saline $(10 \mathrm{~g} \mathrm{NaCl} / \mathrm{l})$ for a period of 21 days, starting with the first day of DOCA treatment. The animals were then followed by weekly measurements of pulse and systolic blood pressure. Systolic blood pressure was recorded using a noninvasive tail-cuff system (Panlab). After 3 weeks of treatment, 


\section{Table 1}

List of primer sequences used

\begin{tabular}{|c|c|c|}
\hline Primer name & Application & Nucleotide sequence \\
\hline Myl9 P1 for & ChIP & 5'-CAGCCCTTGTCTTTTTGTCA-3' \\
\hline Myl9 P1 rev & ChIP & 5'-AACCTATGGCCTCCTACTTG-3' \\
\hline Myl9 P2 for & ChIP & 5'-GTCTCAAAACCCACCCCCACAGT-3' \\
\hline Myl9 P2 rev & ChIP & 5'-GGCTTTTAATTTATGACCTAT-3' \\
\hline Myl9 P3 for & ChIP & 5'-CCCATGGCACCTGCACCTCACC-3' \\
\hline Myl9 P3 rev & ChIP & 5'-CCTGCCTGGCCACATACGAACA-3' \\
\hline Hprt for & RT-PCR & 5'-GCTGGTGAAAAGGACCTCT-3' \\
\hline Hprt rev & RT-PCR & 5'-CACAGGACTAGAACACCTGC-3' \\
\hline Myl9 for & RT-PCR & 5'-CGGGATCCCGAGATGGCTTCATTGATA-3' \\
\hline Myl9 rev & RT-PCR & 5'-CGGAATTCCGTTCATCATGCCCTCCAGA-3' \\
\hline$\beta$-Tubulin for & RT-PCR & 5'-GCGACCTGCAGCTGGACCGAATCT-3' \\
\hline$\beta$-Tubulin rev & RT-PCR & 5'-GGGCGAGGGCACCACACTGAAGG-3' \\
\hline Junb for & Genotyping PCR & 5'-CCCGTCTACACCAACCTCAGCAGT-3' \\
\hline Junb rev & Genotyping PCR & 5'-GGGGGCCATGTAAACCT-3' \\
\hline Col1 $\alpha 2$-iCre for & Genotyping PCR & 5'-ACAGATGCCAGGACATCAGGAAC-3' \\
\hline Col1 $\alpha 2$-iCre rev & Genotyping PCR & 5'-ATCAGCCACACCAGACACAGAGA-3' \\
\hline
\end{tabular}

bined at a 10:1 ratio and neutralized by the addition of $0.1 \mathrm{M} \mathrm{NaOH}$. Fibroblasts were added to the hydrated collagen in 96 wells at a density of $2.5 \times 10^{5}$ cells $/ \mathrm{ml}$ and incubated at $37^{\circ} \mathrm{C}$. After 2 hours incubation, gels were gently detached from the plates, and contraction was monitored over time.

Video microscopy and imaging analyses. Long time-lapse recordings of MEFs were collected for analysis of motility and cellular protrusions. Cells were seeded onto 6-well plates (Nunc) at a confluence of $20 \%$. One day later, long time-lapse recordings (5-minute intervals) were performed and results analyzed as described previously (48).

Retroviral gene transfer. The Junb coding sequence was amplified by PCR and inserted into the PMXpie vector (20). The $M y l 9$ coding sequence was obtained from a mouse cDNA library of RZPD (clone no. IRAVP968G0295D6) and cloned into the pMXpie vector. Junb-deficient MEFs were transduced with retroviral vectors as described $(49,50)$.

RT-PCR and $q R T$-PCR or $q P C R$ analysis. Total RNA was isolated from MEFs, VSMCs, or END cells using peqGold RNApure reagent (Peqlab) according to the instructions of the manufacturer. RNA was reverse transcribed using iScript cDNA synthesis kit (Bio-Rad). Quantitative analy-

animals were sacrificed. Heart weights were measured, and blood vessels were prepared and fixed in zinc fixative or snap-frozen for RNA extraction.

Cell cultures. Generation and culture conditions of MEFs, primary mouse fibroblasts, and END cells have been described previously (20, 45). Murine VSMCs were isolated from branches of mouse mesenteric or femoral arteries and cultured in DMEM (Invitrogen) supplemented with $50 \mathrm{U} / \mathrm{ml}$ penicillin, $50 \mu \mathrm{g} / \mathrm{ml}$ streptomycin, and $15 \%$ fetal bovine serum. Cell phenotype was confirmed by anti- $\alpha$-SMA immunofluorescence staining. For all experiments, VSMCs were used at passages 3-4.

VSMCs were cultured on plastic dishes or collagen type I-linked BioFlex elastomers (Flexcell) following standard protocols or the manufacturer's instructions. Application of cyclic stretch $(10 \%, 0.5 \mathrm{~Hz})$ was performed by using a microprocessor-controlled vacuum pump (FX-3000 FlexerCell Strain Unit, Flexcell) as previously described (46).

Analysis of retinal vessels. Murine eyes were enucleated and fixed in $-20^{\circ} \mathrm{C}$ methanol for 30 minutes. Retinas were dissected as previously described and flattened on slides (47), then incubated in PBS containing 0.5\% Triton $\mathrm{X}-100$ and $10 \%$ normal goat serum for 1 hour at room temperature. After a short rinse with PBS, retinas were incubated with the primary antibodies in PBS, $1 \%$ BSA, $10 \%$ normal goat serum, $0.2 \%$ Tween 20 at $4{ }^{\circ} \mathrm{C}$ overnight. The following primary antibodies were used: mouse monoclonal anti$\alpha$-SMA-Cy3 (Sigma-Aldrich), Bandeiraea simplicifolia (BSI) lectin-FITC (1:100, Sigma-Aldrich), rabbit anti-mouse desmin (Abcam), rabbit antimouse NG2 (Chemicon). Retinas were then washed 3 times with PBS containing $0.2 \%$ Tween 20 , for 10 minutes each, and incubated with secondary antibody for 1 hour at room temperature. After another 3 washes, 10 minutes each, retinas were mounted with Dako fluorescent mounting medium. Retinas were scanned with a Zeiss LSM 710 confocal microscope. Retinal vessel diameter was directly measured with Zeiss Zen software.

Scratch wounding. Cells $\left(1.5 \times 10^{5}\right)$ were plated in 6 -well dishes and grown to confluency. The monolayer was wounded by scratching with a pipette tip and photographed. After 8 hours and 18 hours, the wounded area was photographed again, and wound closure was determined using the distance measuring tool of Adobe Photoshop CS software. Cell motility was estimated as the percentage of the initial wound area (at 0 hours) covered by the cells at 8 hours and 18 hours after wounding.

Collagen contraction assay. The assay was performed as described previously (16). Briefly, type I collagen ( $3 \mathrm{mg} / \mathrm{ml}$, Promocell) and 10x DMEM were com- ses of ethidium bromide gel images (gel documentation system, Peqlab) were performed by use of Java-based ImageJ 1.42 open source software (http:// rsb.info.nih.gov/ij/download/). For primer sequences to amplify $\beta$-tubulin, Hprt, and Myl9 cDNA, refer to Table 1. qRT-PCR/qPCR was performed in a LightCycler (Roche Diagnostics) using the QuantiTect SYBR Green realtime RT-PCR kit (QIAGEN) according to the manufacturer's instructions. Fluorescence was monitored (excitation at $470 \mathrm{~nm}$ and emission at $530 \mathrm{~nm}$ ) at the end of the annealing phase (LightCycler F1 channel). Amplification of the 60S ribosomal protein L32 (RPL32) served as an internal standard. Primer sequences used for PCR amplification are listed in Table 1.

Immunoblot analysis. Whole cell extracts or nuclear extracts were prepared as described (45). Protein $(50 \mu \mathrm{g})$ was separated by SDS-PAGE and transferred onto nitrocellulose membrane, and immunodetection was performed using an enhanced chemiluminescence system (PerkinElmer). Densitometric analyses of scanned X-ray films were performed by use of Java-based ImageJ 1.42 open source software. Antibodies used were anti-Junb (1:500, N17, Santa Cruz Biotechnology Inc.), anti-RCC1 (1:500; BD Biosciences), anti-Hsc70 (1:10,000, Stressgene), anti- $\beta$-actin (1:1,000, Santa Cruz Biotechnology Inc.), anti-phospho-MLC-2-S19 (1:1,000, Cell Signaling Technology), anti-MLC-2 (1:1,000, Cell Signaling Technology), anti-FAK (1:1,000, Cell Signaling Technology), anti-phospho-FAK-Y397 (1:1,000, Biosource), and anti-phospho-FAKY576/577 (1:1,000, Cell Signaling Technology).

Immunofluorescence staining. Cells were seeded on gelatin-coated $(1 \%$, 2 hours, $37^{\circ} \mathrm{C}$, Merck) glass coverslips. Cells were fixed in $4 \%$ formaldehyde, permeabilized in $0.2 \%$ Triton X-100, and blocked in $3 \%$ BSA. Cells were incubated at room temperature with primary antibody for 1-2 hours or with phalloidin conjugated with Alexa Fluor 488 dye for 30 minutes. After washing, cells were incubated with secondary antibody and Hoechst 33342 dye for 1 hour and mounted with Mowiol (Calbiochem). A BZ-8000K fluorescence microscope with integrated CCD camera (Keyence) equipped with Plan Apo objectives (Nikon) was used: $20 \times / 0.75 \mathrm{NA}, 60 \times / 1.4 \mathrm{NA}$ oil. Images were captured and processed using a BZ-Analyzer (Keyence) and Adobe Photoshop CS software. Quantification of data was done using Java-based ImageJ 1.42 open source software. Antibodies used were anti-paxillin (1:200, BD Biosciences), anti-phospho-MLC-2-S19 (1:50, Cell Signaling Technology), goat anti-rat-Alexa Fluor 488 (Molecular Probes), goat anti-rabbit-Cy3 (Dianova), and goat 
anti-mouse-Cy3 (Dianova). To stain F-actin, Alexa Fluor 488-conjugated phalloidin (Invitrogen) was used at a 1:100 dilution. Deparaffinized sections of arteries were blocked for 30 minutes at room temperature with $1 \% \mathrm{BSA}$ in $50 \mathrm{mM}$ Tris- $\mathrm{HCl} \mathrm{pH} 7.6$, incubated overnight at $4{ }^{\circ} \mathrm{C}$ with primary antibodies diluted in blocking solution, washed 3 times with TBST, incubated with secondary antibodies diluted in blocking solution for 1 hour in the dark, washed 3 times with TBST, incubated for 10 minutes with Hoechst 33342 dye, and finally mounted with ProLong Antifade (Invitrogen). Antibodies used were rat anti-CD31 (clone MEC 13.3, Santa Cruz Biotechnology Inc., 1:25); rabbit anti- $\alpha$-SMA, rabbit anti-PCNA, and rat anti-calponin (all Abcam, 1:400); rat anti-desmin (Dianova, 1:250); and donkey anti-rat-Cy2 and donkey anti-rabbit-Cy3 (both Dianova, 1:25). Images were taken using an Olympus CKX41 confocal microscope with a UPlanSApo $\times 40 / 0.90$ objective. Images were acquired with an Olympus DSU camera and processed by cellR software.

ChIP assay. A ChIP kit (Millipore) was used according to the manufacturer's instructions as described previously (20). Antibodies used were Junb (c11, Santa Cruz Biotechnology Inc.) and rabbit IgG (Santa Cruz Biotechnology Inc.). qPCR is described above. For primer sequences, refer to Table 1.

Cyclic stretch analysis and perfusion of isolated mouse arteries. The experiment was performed as previously described (51). In brief, animals were sacrificed, and second-order branches of the mesenteric artery were extracted and inserted into the chamber of the culture myograph system (DMT). The arteries were continuously perfused with DMEM medium (Invitrogen) containing $15 \%$ FCS at increasing intravascular pressure levels or with Tyrode's buffer at a constant longitudinal pressure gradient of $20 \mathrm{mmHg}$ with a flow of approximately $0.07 \mathrm{ml} / \mathrm{min}$ and stimulated or not with acetylcholine and norepinephrine (Sigma-Aldrich) at the following concentrations: $10^{-8} \mathrm{M}$, $10^{-7} \mathrm{M}, 10^{-6} \mathrm{M}$, and $10^{-5} \mathrm{M}$. For de-endothelialization, mesenteric artery preparations were perfused for 10 minutes with $\mathrm{CO}_{2}$-independent medium (Invitrogen). De-endothelialization was verified by immunofluorescence double staining for CD31 and $\alpha$-SMA of paraffin-embedded vessel after perfusion and by acetylcholine-induced dilation of the norepinephrinepreconstricted arteries. The pressure response or the active relaxation/ contraction of the blood vessel was determined by measuring the vessel diameter using VediView software (DMT).

Statistics. SD or SEM is indicated by error bars. Unpaired 2-tailed $t$ tests were performed using GraphPad software. For multiple comparisons, the Bonferroni multiple comparisons test (GraphPad Software Inc., InStat version 3.06 [2003]) was applied. A $P$ value less than 0.05 was considered significant.

\section{Acknowledgments}

The authors thank A. Cerwenka and N. Nausch for providing retroviral vectors; A. Strecker, M. Sator-Schmitt, K. Strittmatter, and Ana-Martin Villalba's laboratory, DKFZ, for technical assistance; and J. Hess and H. Laumen for helpful discussions and critical reading of the manuscript. This work was supported by the Deutsche Forschungsgemeinschaft, TR-SFB 23 (projects A3, B2, C5, and C6).

Received for publication November 13, 2009, and accepted in revised form May 5, 2010.

Address correspondence to: Marina Schorpp-Kistner, Division of Signal Transduction and Growth Control (A100), German Cancer Research Center (DKFZ), DKFZ-ZMBH Alliance, Im Neuenheimer Feld 280, 69120 Heidelberg, Germany. Phone: 49.6221.42.4575; Fax: 49.6221.42.4554; E-mail: marina.schorpp@dkfz.de.
1. de Wit C, Wolfle SE. EDHF and gap junctions: important regulators of vascular tone within the microcirculation. Curr Pharm Biotechnol. 2007;8(1):11-25.

2. Owens GK, Kumar MS, Wamhoff BR. Molecular regulation of vascular smooth muscle cell differentiation in development and disease. Physiol Rev. 2004;84(3):767-801.

3. Gaddam KK, Verma A, Thompson M, Amin R, Ventura $\mathrm{H}$. Hypertension and cardiac failure in its various forms. Med Clin North Am. 2009;93(3):665-680.

4. Nishimura J, Bi D, Kanaide H. Dependence of proliferating dedifferentiated vascular smooth muscle contraction on Rho-Rho kinase system. Trends Cardiovasc Med. 2006;16(4):124-128.

5. Jain RK. Molecular regulation of vessel maturation. Nat Med. 2003;9(6):685-693.

6. Pellegrin S, Mellor H. Actin stress fibres. J Cell Sci. 2007;120(pt 20):3491-3499.

7. Franke RP, Grafe M, Schnittler H, Seiffge D, Mittermayer C, Drenckhahn D. Induction of human vascular endothelial stress fibres by fluid shear stress. Nature. 1984;307(5952):648-649.

8. Fukata Y, Amano M, Kaibuchi K. Rho-Rho-kinase pathway in smooth muscle contraction and cytoskeletal reorganization of non-muscle cells. Trends Pharmacol Sci. 2001;22(1):32-39.

9. Miwa K, Fujita M, Sasayama S. Recent insights into the mechanisms, predisposing factors, and racial differences of coronary vasospasm. Heart Vessels. 2005; 20(1):1-7.

10. Maupin P, Phillips CL, Adelstein RS, Pollard TD. Differential localization of myosin-II isozymes in human cultured cells and blood cells. J Cell Sci. 1994; 107(pt 11):3077-3090.

11. Sandquist JC, Swenson KI, Demali KA, Burridge $\mathrm{K}$, Means AR. Rho kinase differentially regulates phosphorylation of nonmuscle myosin II isoforms $\mathrm{A}$ and $\mathrm{B}$ during cell rounding and migration. J Biol Chem. 2006;281(47):35873-35883.
12. RidleyAJ, et al. Cell migration: integrating signals from front to back. Science. 2003;302(5651):1704-1709.

13. Sanders LC, Matsumura F, Bokoch GM, de Lanerolle P. Inhibition of myosin light chain kinase by p21-activated kinase. Science. 1999;283(5410):2083-2085.

14. Medjkane S, Perez-Sanchez C, Gaggioli C, Sahai E, Treisman R. Myocardin-related transcription factors and SRF are required for cytoskeletal dynamics and experimental metastasis. Nat Cell Biol. 2009; 11(3):257-268.

15. Kajanne R, et al. EGF-R regulates MMP function in fibroblasts through MAPK and AP-1 pathways. J Cell Physiol. 2007;212(2):489-497.

16. Florin L, et al. Delayed wound healing and epidermal hyperproliferation in mice lacking JunB in the skin. J Invest Dermatol. 2006;126(4):902-911.

17. Ahn JD, et al. Inhibitory effects of novel AP-1 decoy oligodeoxynucleotides on vascular smooth muscle cell proliferation in vitro and neointimal formation in vivo. Circ Res. 2002;90(12):1325-1332.

18. Abdel-Malak NA, Srikant CB, Kristof AS, Magder SA, Di Battista JA, Hussain SN. Angiopoietin-1 promotes endothelial cell proliferation and migration through AP-1-dependent autocrine production of interleukin-8. Blood. 2008;111(8):4145-4154.

19. Schmidt D, et al. Critical role for NF-kappaBinduced JunB in VEGF regulation and tumor angiogenesis. EMBO J. 2007;26(3):710-719.

20. Licht AH, et al. JunB is required for endothelial cell morphogenesis by regulating core-binding factor beta. J Cell Biol. 2006;175(6):981-991.

21. Angel P, Karin M. The role of Jun, Fos and the AP-1 complex in cell-proliferation and transformation. Biochim Biophys Acta. 1991;1072(2-3):129-157.

22. Eferl R, Wagner EF. AP-1: a double-edged sword in tumorigenesis. Nat Rev Cancer. 2003;3(11):859-868.

23. Hess J, Angel P, Schorpp-Kistner M. AP-1 subunits: quarrel and harmony among siblings. J Cell Sci. 2004;117(pt 25):5965-5973.
24. Schorpp-Kistner M, Wang ZQ, Angel P, Wagner EF. JunB is essential for mammalian placentation. EMBO J. 1999;18(4):934-948.

25. Kenner L, et al. Mice lacking JunB are osteopenic due to cell-autonomous osteoblast and osteoclast defects. J Cell Biol. 2004;164(4):613-623.

26. Schaub MC, Hefti MA, Zuellig RA, Morano I. Modulation of contractility in human cardiac hypertrophy by myosin essential light chain isoforms. Cardiovasc Res. 1998;37(2):381-404.

27. Teunissen BE, Jongsma HJ, Bierhuizen MF. Regulation of myocardial connexins during hypertrophic remodelling. Eur Heart J. 2004;25(22):1979-1989.

28. Amemiya T, Bhutto IA. Retinal vascular changes and systemic diseases: corrosion cast demonstration. Ital J Anat Embryol. 2001;106(2 suppl 1):237-244.

29. Wang JJ, et al. The long-term relation among retinal arteriolar narrowing, blood pressure, and incident severe hypertension. Am J Epidemiol. 2008; 168(1):80-88.

30. Nguyen TT, Wang JJ, Wong TY. Retinal vascular changes in pre-diabetes and prehypertension: new findings and their research and clinical implications. Diabetes Care. 2007;30(10):2708-2715.

31. Prewitt RL, Rice DC, Dobrian AD. Adaptation of resistance arteries to increases in pressure. Microcirculation. 2002;9(4):295-304.

32. Jay PY Pham PA, Wong SA Elson EL. A mechanical function of myosin II in cell motility. J Cell Sci. 1995;108(pt 1):387-393.

33. Kolega J. Cytoplasmic dynamics of myosin IIA and IIB: spatial 'sorting' of isoforms in locomoting cells. J Cell Sci. 1998;111(pt 15):2085-2095.

34. Matsumura F, Ono S, Yamakita Y, Totsukawa G, Yamashiro S. Specific localization of serine 19 phosphorylated myosin II during cell locomotion and mitosis of cultured cells. J Cell Biol. 1998; 140(1):119-129

35. Grandi AM. Hypertensive heart disease: effects 
of lifestyle modifications and antihypertensive drug treatment. Expert Rev Cardiovasc Ther. 2004 2(4):617-625.

36. Messerli FH, Williams B, Ritz E. Essential hypertension. Lancet. 2007;370(9587):591-603.

37. Farsang $C$, et al. Where are we with the management of hypertension? From science to clinical practice. J Clin Hypertens (Greenwich). 2009;11(2):66-73.

38. Sward K, Mita M, Wilson DP, Deng JT, Susnjar M, Walsh MP. The role of RhoA and Rho-associated kinase in vascular smooth muscle contraction. Curr Hypertens Rep. 2003;5(1):66-72.

39. Heasman SJ, Ridley AJ. Mammalian Rho GTPases: new insights into their functions from in vivo studies. Nat Rev Mol Cell Biol. 2008;9(9):690-701.

40. Kogata N, Tribe RM, Fassler R, Way M, Adams $\mathrm{RH}$. Integrin-linked kinase controls vascular wall formation by negatively regulating Rho/ROCKmediated vascular smooth muscle cell contraction. Genes Dev. 2009;23(19):2278-2283.

41. Chua CC, Hamdy RC, Chua BH. Upregulation of endothelin-1 production by lysophosphatidic acid in rat aortic endothelial cells. Biochim Biophys Acta. 1998 1405(1-3):29-34.

42. Eid AH, Chotani MA, Mitra S, Miller TJ, Flavahan NA. Cyclic AMP acts through Rap1 and JNK signaling to increase expression of cutaneous smooth muscle alpha2C-adrenoceptors. Am J Physiol Heart Circ Pbysiol. 2008;295(1):H266-H272.

43. Mao X, Orchard G, Lillington DM, Russell-Jones R, Young BD, Whittaker SJ. Amplification and overexpression of JUNB is associated with primary cutaneous T-cell lymphomas. Blood. 2003;101(4):1513-1519.

44. Zhang W, Hart J, McLeod HL, Wang HL. Differential expression of the AP-1 transcription factor family members in human colorectal epithelial and neuroendocrine neoplasms. Am J Clin Pathol. 2005; 124(1):11-19

45. Andrecht S, Kolbus A, Hartenstein B, Angel P, Schorpp-Kistner M. Cell cycle promoting activity of JunB through cyclin A activation. J Biol Chem. 2002; 277(39):35961-35968.

46. Korff T, Aufgebauer K, Hecker M. Cyclic stretch controls the expression of CD40 in endothelial cells by changing their transforming growth factor-beta 1 response. Circulation. 2007;116(20):2288-2297.

47. Gerhardt H, et al. VEGF guides angiogenic sprouting utilizing endothelial tip cell filopodia. $J$ Cell Biol. 2003;161(6):1163-1177.

48. Yang $Y$, et al. MEGAP impedes cell migration via regulating actin and microtubule dynamics and focal complex formation. Exp Cell Res. 2006; 312(12):2379-2393

49. Cerwenka A, et al. Retinoic acid early inducible genes define a ligand family for the activating NKG2D receptor in mice. Immunity. 2000;12(6):721-727.

50. Nausch N, Florin L, Hartenstein B, Angel P, Schorpp-Kistner M, Cerwenka A. Cutting edge: the AP-1 subunit JunB determines NK cell-mediated target cell killing by regulation of the NKG2D-ligand RAE-1epsilon. J Immunol. 2006;176(1):7-11.

51. Demicheva E, Hecker M, Korff T. Stretch-induced activation of the transcription factor activator protein-1 controls monocyte chemoattractant protein-1 expression during arteriogenesis. Circ Res. 2008; 103(5):477-484. 